\title{
Predicting Escalated Care in Recurrent Wheezing Infants
}

\author{
Jefferson Buendia ${ }^{1}$, Carlos Rodriguez-Martinez ${ }^{2}$, and Ranniery Acuña-Cordero ${ }^{3}$ \\ ${ }^{1}$ University of Antioquia \\ ${ }^{2}$ Universidad Nacional de Colombia \\ ${ }^{3}$ Hospital Militar Central
}

September 5, 2020

\begin{abstract}
Introduction The risk stratification of infants presenting to the emergency department with bronchiolitis who are at risk for receiving airway support during their hospital stay has been insufficiently studied. The aim of this study was to determine the clinical predictors of hospitalization with airway support ("escalated care") among infants with recurrent wheezing evaluated in the emergency department Methods: We conducted a retrospective cohort study in infants with one or more wheezing episode, younger than two years of age in tertiary centers in Rionegro, Colombia. The primary outcome measure was escalated care defined as hospitalization plus any airway support. A multivariate logistic regression model was performed to estimate predictors of escalated care. To assess discrimination and calibration, area-under-the-curve (AUC) and calibration plots were calculated. Results A total of 665 cases were included and 85 infants received escalated care. The variables included risk score for escalated care within 5 days of admission to the emergency room including prematurity, poor feeding, nasal flaring and/or grunting, and previous wheezing episodes requiring hospitalization, The model has a high specificity (99.6\%) with acceptable AUC of 0.70 (CI 95\% 0.60- 0.74). Conclusion: A clinical risk score was created based on the odds ratio of each of the identified variables, which appears to be useful for estimating the absolute risk of escalated care within 5 days of admission to the ED. However, external validation is required before this clinical score is applied in general practice in any ED setting
\end{abstract}

\section{Full title: Predicting Escalated Care in Recurrent Wheezing Infants}

\section{Short running title: Escalated Care in Recurrent Wheezing}

Jefferson Antonio Buendía, MD, MSc, $\mathrm{PhD}^{1}$, Ranniery Acuña-Cordero MD², Carlos E. Rodriguez-Martinez $\mathrm{MD}, \mathrm{MSc}, \mathrm{PhD}(\mathrm{c})^{3,4}$

Department of Pharmacology and Toxicology, School of Medicine, Pharmacology and Toxicology Research Group (INFARTO). Universidad de Antioquia, Medellín, Colombia.

Department of Pediatric Pulmonology, Hospital Militar Central, Departamento de Pediatría, Facultad de Medicina, Universidad Militar Nueva Granada, Bogotá, Colombia

Department of Pediatrics, School of Medicine, Universidad Nacional de Colombia, Bogota, Colombia

Department of Pediatric Pulmonology and Pediatric Critical Care Medicine, School of Medicine, Universidad El Bosque, Bogota, Colombia

Corresponding autor: Jefferson A. Buendía, MD, Ph.D., Facultad de Medicina, Universidad de Antioquia, Carrera 51D \#62-29, Tel: +574 2196046; Medellín, Colombia. E-mail: jefferson.buendia@gmail.com. E-mail: JAB: jefferson.buendia@gmail.com, 
Author's contributions: JAB, RAC, CERM participated in designing the work and the acquisition, analysis, and interpretation of data. All authors approve the version to be published.

Source(s) of support: own funds

Word count: 2327

Summary: There are no scales that predict the risk of ventilatory support in recurrent wheezing. Our scale allows us to determine those at high risk of ventilatory support with high accuracy and to act early to improve health outcomes.

\section{ABSTRACT}

\section{Introduction}

The risk stratification of infants presenting to the emergency department with bronchiolitis who are at risk for receiving airway support during their hospital stay has been insufficiently studied. The aim of this study was to determine the clinical predictors of hospitalization with airway support ("escalated care") among infants with recurrent wheezing evaluated in the emergency department

\section{Methods :}

We conducted a retrospective cohort study in infants with one or more wheezing episode, younger than two years of age in tertiary centers in Rionegro, Colombia. The primary outcome measure was escalated care defined as hospitalization plus any airway support. A multivariate logistic regression model was performed to estimate predictors of escalated care. To assess discrimination and calibration, area-under-the-curve (AUC) and calibration plots were calculated.

\section{Results}

A total of 665 cases were included and 85 infants received escalated care. The variables included risk score for escalated care within 5 days of admission to the emergency room including prematurity, poor feeding, nasal flaring and/or grunting, and previous wheezing episodes requiring hospitalization, The model has a high specificity $(99.6 \%)$ with acceptable AUC of 0.70 (CI 95\% 0.60- 0.74).

\section{Conclusion :}

A clinical risk score was created based on the odds ratio of each of the identified variables, which appears to be useful for estimating the absolute risk of escalated care within 5 days of admission to the ED. However, external validation is required before this clinical score is applied in general practice in any ED setting.

Keywords: bronchiolitis, asthma, risk, prediction rule

\section{Introduction}

Recurrent wheezing is very prevalent among infants. Approximately one third of children experience wheezing at least once by the time they reach 3 years of age(1). This has a high impact on society due to the elevated utilization of ED visits and hospitalizations(2).

Among the aspects that most impact the morbidity of patients is the early identification of patients who may develop or require mechanical ventilation; the improper delay of mechanical ventilation can increase the mortality rate(3). As reported in the literature, 5 - $43 \%$ of patients failed oxygen support or non-invasive pressure-positive ventilation and subsequently required mechanical ventilation $(4,5)$. If one can predict which patients will require different oxygenation systems until they reach mechanical ventilation, patients can be put on mechanical ventilation immediately (3). This prediction would be made before a patient is put on low system oxygen therapy or non-invasive pressure-positive ventilation. Models have been developed that predict the use of oxygen or the use of non-invasive ventilation or mechanical ventilation, all separately (6). This risk stratification has been insufficiently studied. In a retrospective cohort study in infants aged $<12$ months with bronchiolitis, Freire et al. identified the following as predictors of received escalated care: 
oxygen saturation $<90 \%$, nasal flaring and/or grunting, apnea (OR: 3.0[95\% CI 1.9-4.8]), retractions, age [?]2 months, dehydration, and poor feeding. However, this study only included patients in their first wheezing episode and without any comorbidities; which limits its use since the majority of patients who receive just escalation are those who have more than one wheezing episode and have comorbidities $(3,7)$. The aim of this study was to determinate the clinical predictors of hospitalization with airway support ("escalated care") among infants with recurrent wheezing evaluated in an emergency department.

\section{Methods}

We performed a retrospective cohort study that included all infants with one or more wheezing episodes who were younger than two years old in tertiary centers in Rionegro, Colombia, between January 2019 and December 2019. The municipality of Rionegro had a total population of 101,046 inhabitants, with two tertiary referral hospitals(8). Inclusion criteria were defined as children younger than two years of age admitted to the pediatric ward diagnosed with a wheezing episode. Patients without lower respiratory compromises, with positive bacterial cultures on admission or a confirmed whooping cough (culture or PCR) were excluded. The study protocol was approved by the Institutional Review Board of the University of Antioquia (No 18/2015).

\section{Procedures}

Medical records of all patients accepted to the emergency department were reviewed. We collected the following variables: age, sex, weight, height, signs, and symptoms on admission (including fever, chest in-drawing, chest auscultation, \%SpO2), vaccination scheduled chart for age, current exposure (maternal or paternal) to cigarette smoking, history of prematurity and bronchopulmonary dysplasia confirmed by a neonatologist upon discharge from NICU, comorbidities (congenital heart disease, neurological disease), diagnostic tools such as chest X-rays, etc. In our hospitals, bronchodilators and systemic steroids are used at the discretion of attending physicians according to national clinical bronchiolitis guidelines(9). Nasopharyngeal aspirate was taken immediately upon admission to the emergency department within 48 hours of admission using a standard technique. RSV was confirmed using direct immunofluorescence (Light Diagnostics TM Respiratory Panel 1 DFA, Merck-Millipore Laboratory).

\section{Outcome definition}

Our primary outcome was escalated care, defined as hospitalization plus any of the following: HFNC, noninvasive ventilation (e.g., continuous or biphasic positive airway pressure), intubation and ventilation, or management in the ICU without airway support. The criteria for ICU admission were impair hypoxemia or hypercapnia, respiratory distress, inspired fraction of oxygen of over fifty percent, hemodynamic instability, or apnea.

\section{Sample size}

Estimates were made according to Riley's recommendations for estimating sample sizes for multivariate predictive models with binary outcomes (10). Assuming 4 potential predictor variables, escalated care prevalence of $9.6 \%$ (11), proportion of unexplained variation of 0.15 (recommended by Riley in the absence of good-quality prior information), shrinkage of 0.9 , marginal error in estimating the intercept of 0.05 , and an acceptable difference between the apparent and adjusted R2 of 0.05 , the sample size required for the development of this new model was 700 patients or 68 events.

\section{Statistical analysis}

Continuous variables were shown as mean +- standard deviation (SD) or median (interquartile range [IQR]), whichever was appropriate. Categorical variables were shown as numbers (percentage). To identify factors independently associated with escalated care, we used logistic regression and estimated odds ratios (ORs) and adjusted for potential confounding variables.

Initially, the regression model only included variables associated with escalated care with values of $\mathrm{p}<0.2$ or that changed the effect estimate by over $10 \%$ after their inclusion. A multivariate logistic regression model 
was performed with a backward elimination method, used with a p value of 0.05 as the limit value for the model entry. The variable selection and modeling processes were made following the recommendations of Greenland(12). The method of Sullivan et al. was used to generate the risk scores and estimate the risks observed with each score (13). To assess discrimination, area-under-the-curve (AUC) was estimated with a 95\% confidence interval and plotted using AUC-ROC plots(14). To correct prediction probabilities for overoptimistic predictions and to evaluate the calibration, the model was analyzed by comparing the predicted probability to the observed probability of mortality and examined with a calibration plot and calibration slope with 95\% CI. Calibration plots (STATA function: pmcalplot) displayed observed risk by deciles of the predicted risk and examined risk at the individual level using Locally Weighted Scatterplot Smoothing algorithms(15). We also calculated the Hosmer - Lemeshow goodness of fit test as well as calibration curves between the predicted probabilities and the observed data. To correct sampling bias of variance parameters and to evaluate the internal validity of the model, repeated curved validation "tenfold cross-validation" were used, comparing the area under the ROC curve obtained in the repetitions with that observed in the model $(15,16)$; as well as with the bootstrapping technique with which both the adjusted oppressiveness and area values were estimated. All statistical tests were two-tailed, and the significance level used was $p<0.05$. The data were analyzed with Statistical Package Stata 15.0 (Stata Corporation, College Station, TX).

\section{Results}

\section{Study population}

During the study period, 665 cases were included. A total of 85 infants received escalated care, of which $34(40 \%)$ were treated with HFNC, $2(2.4 \%)$ received non-invasive ventilation, $6(7.6 \%)$ were mechanically ventilated, and $43(50 \%)$ received conventional oxygen therapy. The characteristics of the infants who did and did not receive escalated care and the bivariable associations between postulated predictors and escalated care appear in Table 1.

\section{Multivariable Analysis}

The variables included in the multivariable analysis included prematurity, poor feeding, nasal flaring and/or grunting and previous wheezing episodes requiring hospitalization. The risk points assigned to each predictor variable appear in Table 2. The total risk score ranged from 0 to 10 points. The overall clinical risk score for escalated care and estimated absolute percent risk of escalated care within 5 days of admission to the emergency room appear in Table 3.

The model have a sensitivity of $7 \%$, specificity of $99.6 \%$, positive predictive value of $1 \%$ and negative predictive value of $92.94 \%$, a percentage of cases correctly classified of $87.8 \%$ with AUC for the risk score was 0.728, Figure 1. Figure 1 and 2 show corrected average AUC and calibration plots (slope= 1.09). The tenfold cross-validation revealed a corrected average AUC of 0.70 (CI 95\% 0.60-0.74) with mean overoptimism obtained by a bootstrapping of $0.068 \%$ (Standard error: 0.0031 ). The risk score model had an excellent calibration and goodness of fit through the Hosmer-Lemeshow test $(\mathrm{p}=0.47)$.

\section{Discussion}

The main purpose of this study was to determinate the clinical predictors of hospitalization with airway support ("escalated care") among infants with recurrent wheezing evaluated in the emergency department. Our study shows that prematurity, poor feeding, nasal flaring and/or grunting and previous wheezing were independent predictors of escalated care. The clinical risk score, with a demonstrated high stability and discrimination ability, derived in a non-selected population is used to quantify estimated risk for escalated care in patients with recurrent wheezing during the hospital stay.

Most studies have been focused on predicting hospital admission from the ED or PICU, or using a specific type of NIPPV or mechanical ventilation (6). We have focused our outcome on receipt of escalated care, since these infants needs timely identification due to the risk of developing acute respiratory failure. The clinical use of the risk score must be prospectively validated; it has the potential to individualize recurrent wheezing treatment. 
Research of predictive models in infants with bronchiolitis has generally been focused on inpatients, especially infants admitted to PICUs, with relatively small numbers of patients, and only focused on RSV bronchiolitis (6). In a retrospective cohort study with 2722 infants conducted by Freire el al., around 261 $(9.6 \%)$ received escalated care. Multivariable predictors of escalated care were oxygen saturation, nasal flaring and/or grunting, apnea, retractions, age [?]2 months, dehydration, and poor feeding. However, this study was conducted in children younger than 12 months with their first wheezing episode and excluded infants with comorbidities, limiting their external validity to other subpopulations. Through a non-selected population-based cohort study of 34,270 infants in Ontario, Schun et al. identified the following as predictors of admission: critical care comorbidities (OR 5.33; 95\% CI 2.82-10.10), younger age [months] (OR 1.47; 95\% CI 1.33-1.61), low income (OR 1.53; 95\% CI 1.01-2.34), younger gestational age [weeks] (OR 1.14; 95\% CI 1.06-1.22), and emergent presentation (Canadian Triage and Acuity Scale 2) at the index visit (OR 1.55, 95\% CI 1.03-2.33). The odds of these outcomes with comorbidities plus [?]2 other predictors were 25 times higher than in infants without predictors (OR 25.1, 95\% CI 11.4-55.3) (16). The differences between the risk factors found in these studies compared to our study are due to the differences in the populations studied. While our study included patients with or without comorbidities and who had more than one wheezing episode, Freire's study focused on low-risk patients in their first wheezing episode. In this sense, our study is complementary to Freire's study, indicating that variables such as nasal flutter and poor feeding that this study found as predictors also continue to be predictors in recurrent wheezing patients with comorbidities. The aim of our tool is for it to be used by clinicians to guide management decisions. For example, the score would support the outpatient management of premature patients with wheezing without respiratory distress issues or with adequate feeding. Around $25 \%$ of hospitalized infants with bronchiolitis receive no evidence-based therapies (17), and the use of the risk score may result in a lower hospitalization rate and lower health-care expenditure. Our risk score employs clinical items in routine use for assessing bronchiolitis.

Our study has limitations. Firstly, since this study was based on a review of medical records, we cannot include other variables such as environmental pollution and genetic factors, and residual confounding cannot be excluded. Secondly, the study was conducted in a tertiary referral hospital, and therefore the patients included represent the high spectrum of severity, limiting the generalization of results to other contexts. However, the similarity of our population in terms of clinical characteristics, risk factors, and seasonality of bronchiolitis in our country with previous reports suggests strength and consistency in our results $(18,19)$. Thirdly, in our study, we used an immunofluorescent assay to diagnose RSV infections. Although this is widely available and easy to perform, we did not determine the RSV genomic load, and we also did not test for viruses. This may generate a differential misclassification bias, which could have overestimated the true association between RSV isolation and the outcome variable; however, the previous evidence in other populations had confirmed this association being plausible in our results.

\section{Conclusion :}

In conclusion, the present study shows that prematurity, poor feeding, nasal flaring and/or grunting, and more than one previous episode of wheezing requiring hospitalization are independent predictors of hospitalization with airway support ("escalated care") in a population of infants with recurrent wheezing attended to in the ED. A clinical risk score was created based on the odds ratio of each of the identified variables, which appears to be useful for estimating the absolute risk of escalated care within 5 days of admission to the ED; however, external validation is required before this clinical score is applied in general practice in any ED setting.

\section{Abbreviations :}

CHD: Congenital heart disease

BPD: Bronchopulmonary dysplasia

PICU: pediatric intensive care unit admission

RSV: Respiratory syncytial virus 
NIPPV: non-invasive positive-pressure ventilation

ED: emergency department

\section{Declarations}

Ethics approval: The Universidad de Antioquia's Medicine Faculty Ethics Committee reviewed and approved the protocol before any procedure was undertaken

Consent for publication: All authors consent to the publication of this paper

Competing interests. None declared.

Funding: Own funding

Availability of Data and Materials: None

Acknowledgments: None declared.

\section{Bibliography}

1. Martinez FD. Trends in asthma prevalence, admission rates, and asthma deaths. Respir Care. 2008;53(5):561-5; discussion 5-7.

2. Graham LM. Preschool wheeze prognosis: how do we predict outcome? Paediatr Respir Rev. 2006;7 Suppl 1:S115-6.

3. Najaf-Zadeh A, Leclerc F. Noninvasive positive pressure ventilation for acute respiratory failure in children: a concise review. Ann Intensive Care. 2011;1(1):15.

4. Javouhey E, Barats A, Richard N, Stamm D, Floret D. Non-invasive ventilation as primary ventilatory support for infants with severe bronchiolitis. Intensive Care Med. 2008;34(9):1608-14.

5. Abboud PA, Roth PJ, Skiles CL, Stolf A, Rowin ME. Predictors of failure in infants with viral bronchiolitis treated with high-flow, high-humidity nasal cannula therapy*. Pediatr Crit Care Med. 2012;13(6):e343-9.

6. Luo G, Nkoy FL, Gesteland PH, Glasgow TS, Stone BL. A systematic review of predictive modeling for bronchiolitis. Int J Med Inform. 2014;83(10):691-714.

7. Mayordomo-Colunga J, Medina A, Rey C, Diaz JJ, Concha A, Los Arcos M, et al. Predictive factors of non invasive ventilation failure in critically ill children: a prospective epidemiological study.Intensive Care Med. 2009;35(3):527-36.

8. Estadisticas DAN. Proyecciones de poblacion 2018 [03/07/2019]. Available from: https://www.dane.gov.co/index.php/estadisticas-por-tema/demografia-y-poblacion/proyecciones-depoblacion.

9. Colciencias MdSyPS-. Guia de Practica Clinica Para el diagnostico, atencion integral y seguimiento de ninos y ninas con diagnostico de Asma. Guia No. 012014 [Available from: ,http://gpc.minsalud.gov.co/gpc-sites/Repositorio/Conv_500/GPC_asma/GPC_Comp_Asma.pdf.

10. Riley RD, Ensor J, Snell KIE, Harrell FE, Jr., Martin GP, Reitsma JB, et al. Calculating the sample size required for developing a clinical prediction model. BMJ. 2020;368:m441.

11. Papenburg J, Fontela PS, Freitas RR, Burstein B. Inappropriate Antibiotic Prescribing for Acute Bronchiolitis in US Emergency Departments, 2007-2015. J Pediatric Infect Dis Soc. 2019. 
12. Greenland S. Modeling and variable selection in epidemiologic analysis. Am J Public Health. 1989;79(3):340-9.

13. Sullivan LM, Massaro JM, D'Agostino RB, Sr. Presentation of multivariate data for clinical use: The Framingham Study risk score functions. Stat Med. 2004;23(10):1631-60.

14. Altman DG, Bland JM. Diagnostic tests 3: receiver operating characteristic plots. BMJ. 1994;309(6948):188.

15. Steyerberg EW, Vickers AJ, Cook NR, Gerds T, Gonen M, Obuchowski N, et al. Assessing the performance of prediction models: a framework for traditional and novel measures. Epidemiology. 2010;21(1):12838.

16. Schuh S, Kwong JC, Holder L, Graves E, Macdonald EM, Finkelstein Y. Predictors of Critical Care and Mortality in Bronchiolitis after Emergency Department Discharge. J Pediatr. 2018;199:217-22 e1.

17. Buendia JA, Patino DG. Costs of Respiratory Syncytial Virus Hospitalizations in Colombia. Pharmacoecon Open. 2020.

18. Rodriguez-Martinez CE, Sossa-Briceno MP, Castro-Rodriguez JA. Direct medical costs of RSV-related bronchiolitis hospitalizations in a middle-income tropical country. Allergol Immunopathol (Madr). 2019.

19. Rodriguez-Martinez CE, Sossa-Briceno MP, Nino G. Predictors of prolonged length of hospital stay for infants with bronchiolitis. J Investig Med. 2018;66(6):986-91.

Figure legends-

Figure 1. Receiver operating characteristic curve for the clinical risk score.

Figure 2. Calibration plots for the clinical risk score.

\section{Hosted file}

Table 1.docx available at https://authorea.com/users/316889/articles/478421-predictingescalated-care-in-recurrent-wheezing-infants

\section{Hosted file}

Table 2.docx available at https://authorea.com/users/316889/articles/478421-predictingescalated-care-in-recurrent-wheezing-infants

\section{Hosted file}

Table 3.docx available at https://authorea.com/users/316889/articles/478421-predictingescalated-care-in-recurrent-wheezing-infants 

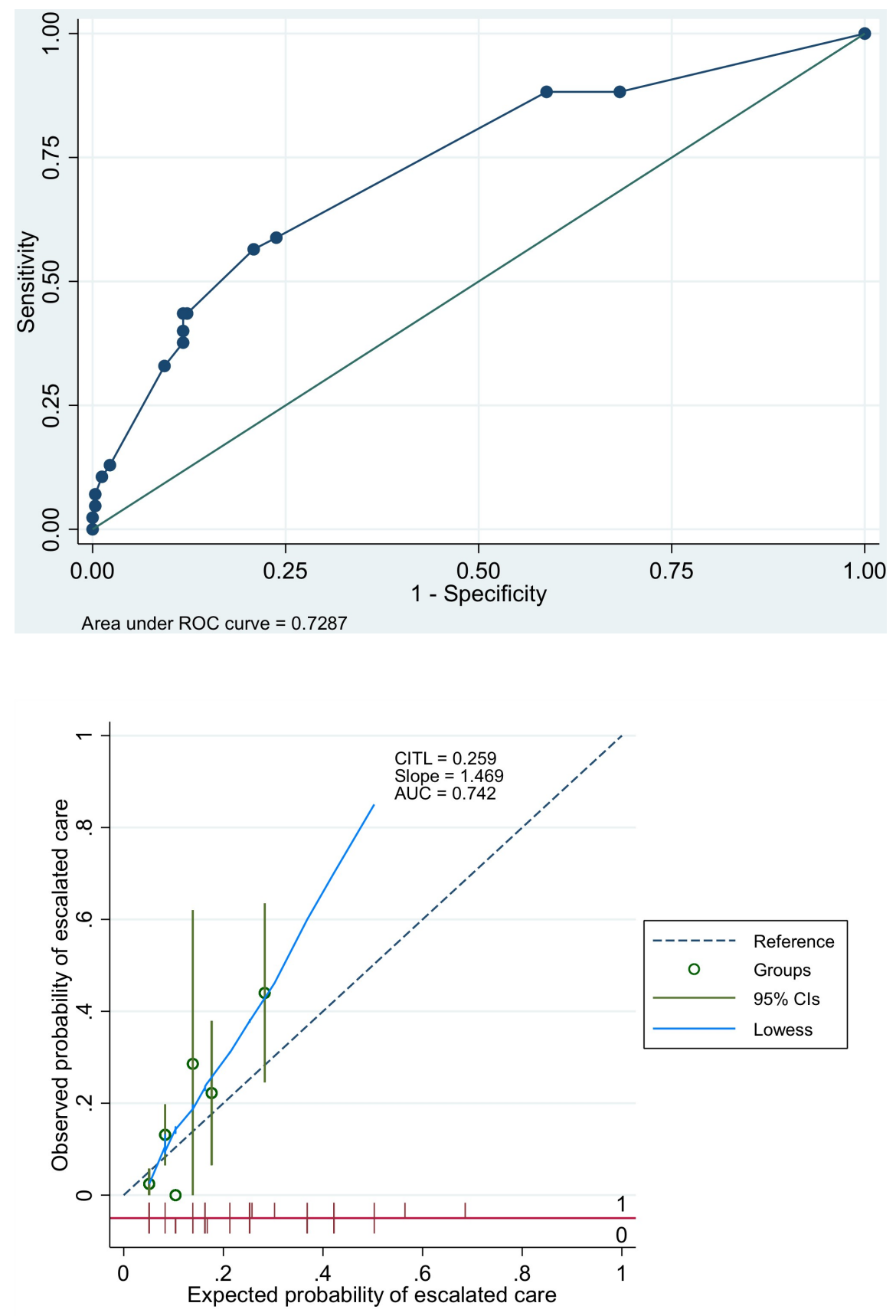